\title{
ORFEUS-SPAS:
}

\section{THE BERKELEY EUV SPECTROMETER}

\author{
STUART BOWYER \\ Space Astrophysics Group, Center for EUV Astrophysics, \\ University of California, Berkeley, California 94720 \\ and \\ MARK HURWITZ \\ Space Astrophysics Group, Center for EUV Astrophysics, \\ University of California, Berkeley, California 94720
}

\section{Introduction}

ORFEUS-SPAS, the Orbiting Retrievable Far and Extreme Ultraviolet Spectrometers, on the Astro-SPAS space platform, is a joint project of NASA and the BMFT in the Federal Republic of Germany (see Kraemer et al. 1990, this volume). The Berkeley spectrograph for this mission will obtain high-resolution $(\lambda / \Delta \lambda>7000)$ spectra of point sources between 390 and $1200 \AA$. The Berkeley instrument incorporates a set of four novel spherically figured, varied line-space (SVLS) gratings used in a geometry that is similar to that of the classic Rowland mount to span this large wavelength interval. Two spectral detector units containing curved MCPs and delay-line anodes encode the arriving photons in digital format for telemetry. An additional optic directs the image of the source in the entrance aperture onto a sealed FUV detector which is used to track the source as it drifts during an observation, enabling a post-flight reconstruction of the spacecraft pointing vector. This in turn will allow us to define the wavelength of each recorded photon with precision.

\section{The Berkeley Spectrometer}

In Figure 1 we show a schematic layout of the optical components of the spectrograph. The instrument contains four diffraction gratings, arranged so that each is illuminated by a "wedge" on the annular aperture of the telescope primary mirror. Each wedge subtends about $20 \%$ of the available geometrical area of the $1 \mathrm{~m}$ diameter primary and therefore has a focal ratio of about $f / 5$ rather than the full $f / 2.4$ from the primary mirror. Each grating is used to study only one-fourth of the total 390-1200 $\AA$ bandpass, and operates over a relatively small range of diffraction angle $\beta$, allowing high diffraction efficiency to be maintained. Two spectral detector units each accept the spectra from two diffraction gratings.

In our design, each diffraction grating in the instrument is spherical, with a radius of curvature of $1 \mathrm{~m}$. Unlike the conventional Rowland spectrograph, the groove spacing of each grating varies as a fourth order function of position on the optic. Furthermore the distances from the telescope image to the grating center, and from

Y. Kondo (ed.), Observatories in Earth Orbit and Beyond, 475-480.

(C) 1990 Kluwer Academic Publishers. Printed in The Netherlands. 


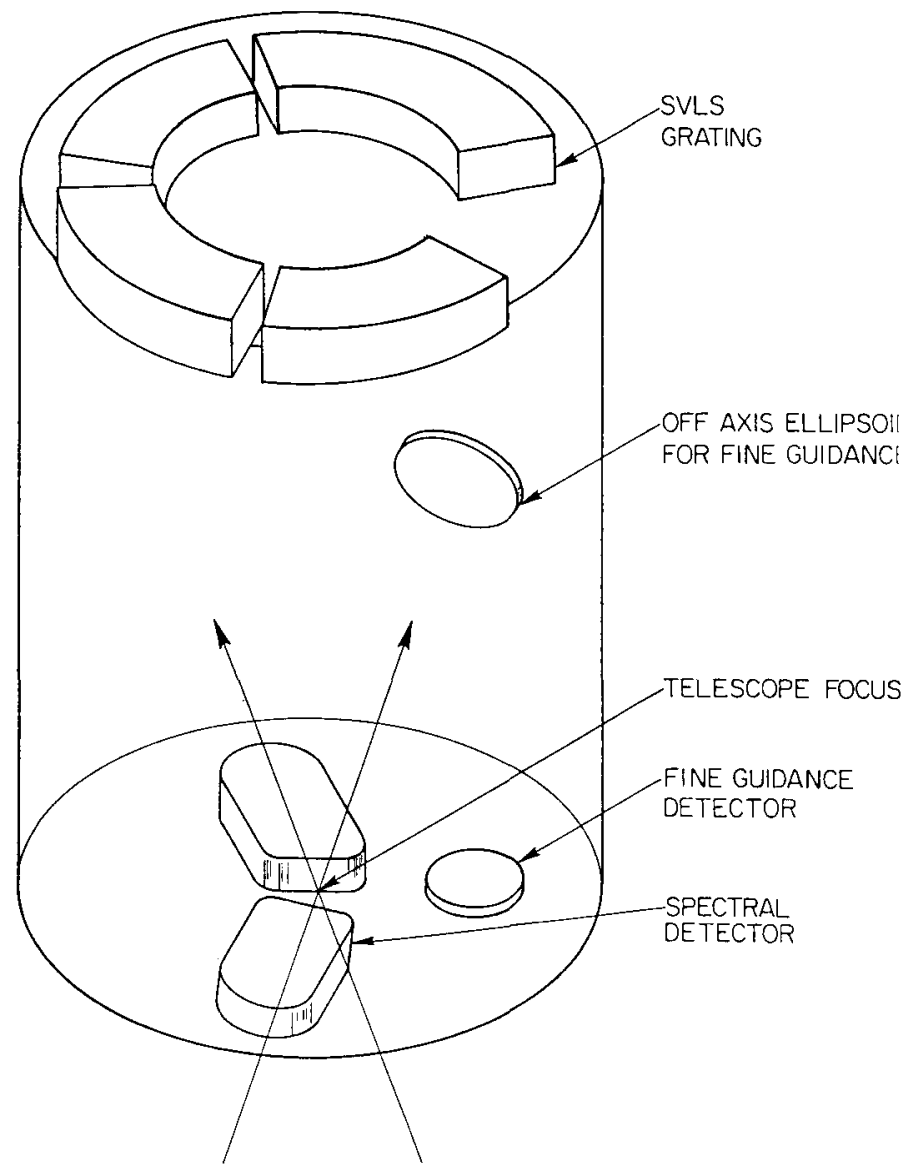

Fig. 1. Schematic layout of the Berkeley spectrograph.

the grating center to the detector surface, are not equal to the values on the corresponding Rowland circle. The astigmatism and aberrations of this spectrograph are substantially lower than what would be achievable with a conventional Rowland spectrograph and toroidal, uniform line-space diffraction gratings. A more detailed description of SVLS spectrometers can be found in Harada and Kita (1980).

Two detectors are used, each accepting the dispersed spectra from two diffraction gratings. The MCPs are spherically curved, with a radius chosen to provide a close match to the tangential focal surface of the diffraction gratings. A flat detector would have limited the resolution to $\sim 1500$. Each detector contains a stack of two MCPs, the electron charge clouds from which strike a delay-line anode readout system (Lampton, Siegmund and Raffanti 1987).

The fine guidance system consists of a small off-axis ellipsoid, which intercepts a fraction of the beam from the primary mirror and images the target onto a sealedtube MCP detector equipped with a wedge-and-strip readout system (Martin et al. 1981). The fine guidance system is sensitive to photons in the $1400-1800 \AA$ 


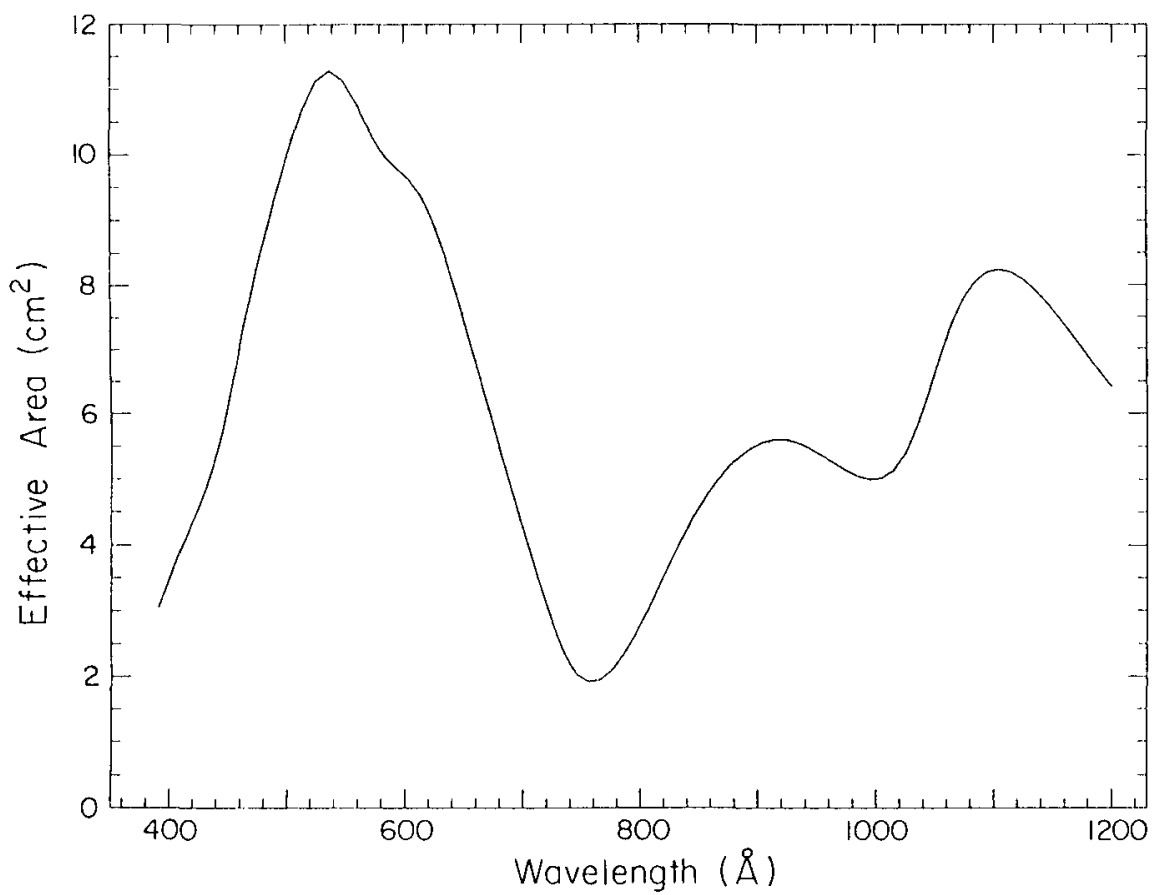

Fig. 2. Predicted effective area of the instrument vs. wavelength. Fabricated instrument will display small discontinuities at crossover wavelengths between gratings due to variations in diffraction efficiency and coating reflectivity.

band. Each pixel on the detector represents about $3^{\prime \prime}$ on the sky. To determine the location of the source to a precision of about $1^{\prime \prime}$, we will place the detector so that the image is defocused to cover $\sim 3$ pixels and calculate a centroid of the photons recorded during a given integration period. The integration period is determined by the expected drift rate of the target in the aperture; during one second the target should move no more than about $1^{\prime \prime}$. Even a relatively faint target such as the hot WD HZ 43 should produce over 100 events $\mathrm{s}^{-1}$ on the detector, enabling a centroid to be calculated to an accuracy better than $1^{\prime \prime}$.

\section{Instrument Performance}

The telescope primary mirror will be coated with iridium, a stable coating which provides good normal-incidence reflectivity across the instrument bandpass. The diffraction gratings will be coated with the same material. We expect that the gratings will achieve a groove efficiency of $\sim 50 \%$, and that the quantum detection efficiency will be equal to that measured for the stable photocathode KBr by Siegmund et al. (1988). The resulting curve of effective-area-versus-wavelength is shown in Figure 2.

Prior to the launch of ORFEUS in 1992, NASA will have flown two other major instruments with spectroscopic capabilities in the EUV. These are HUT (Davidsen 


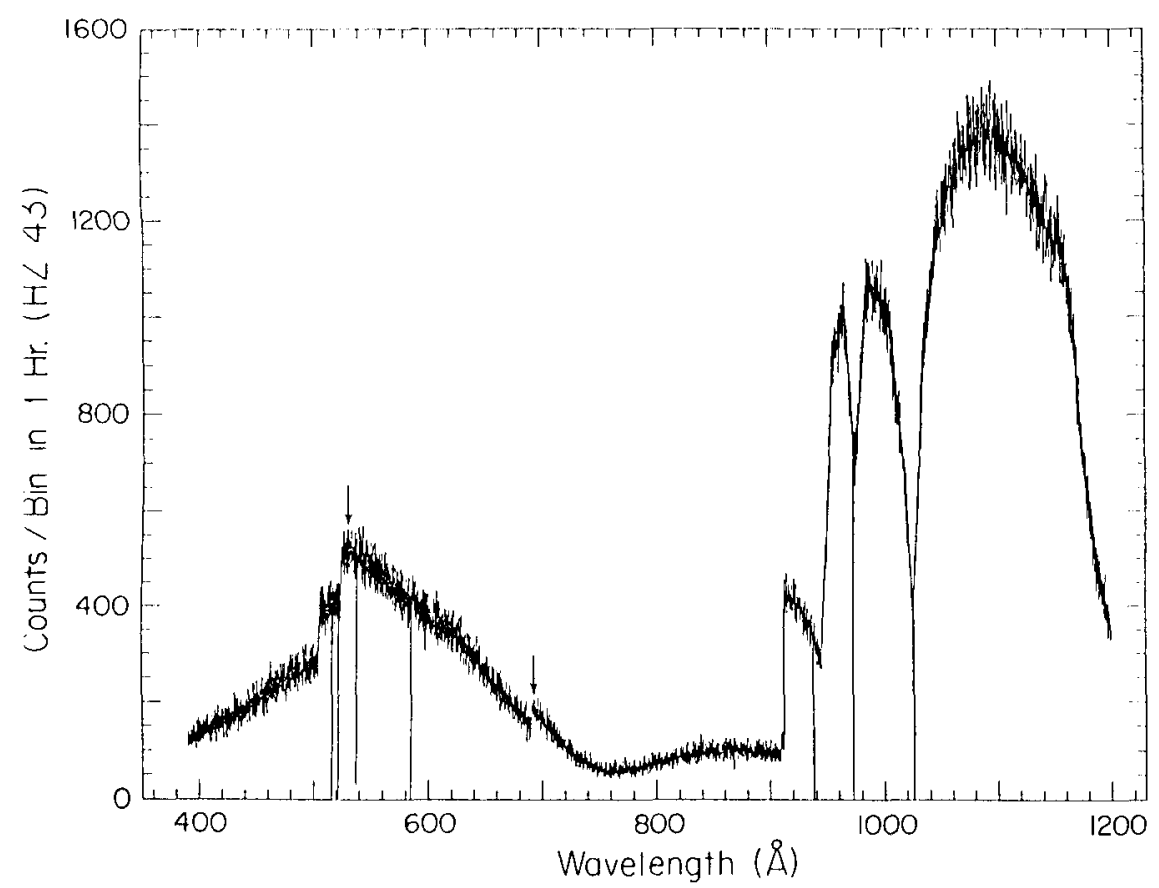

Fig. 3. Sample spectrum of hot WD HZ 43. Counts per spectral bin for a 3600 s integration are plotted vs. wavelength. Column density of $\mathrm{H}$ I assumed to be $2 \times 10^{17} \mathrm{~cm}^{-2}$; column of He I assumed to be 1/10 that of H I. Absorption edges of interstellar H I and He I are shown, as are strong resonance lines of these species. Because the spectral resolution changes discontinuously at grating crossover wavelengths, the simulated counts per spectral bin display those discontinuities (marked with an arrow).

et al. 1989) and EUVE (Malina and Bowyer 1989). Although the relative sensitivity of these instruments depends on quantities that may be difficult to estimate (e.g., the detector background rate), for relatively bright sources it is the effective area that limits the achievable signal-to-noise, so it is the effective area - and spectral resolution - that we compare here.

HUT contains a normal-incidence mirror which is only slightly smaller than that of ORFEUS. The spectrograph design is a classical Rowland mount. At wavelengths between 840 and $1580 \AA$, the HUT grating is used in first order and should achieve a net effective area that is $\sim 1.5$ times that of ORFEUS. Between 420 and $925 \AA$, the HUT grating is used in second order and can achieve an effective area roughly equal to that of ORFEUS. But because many targets will be substantially fainter in that band than at first-order wavelengths, an aluminum filter will certainly be needed for continuum measurements to attenuate the first-order radiation and may well be needed for studies of line radiation. This filter reduces the HUT effective area at the short wavelengths to $1 / 6$ to $1 / 10$ that of ORFEUS, depending on wavelength. The spectral resolution of $H U T$ is $\sim 400$, approximately $1 / 20$ that of ORFEUS. The 


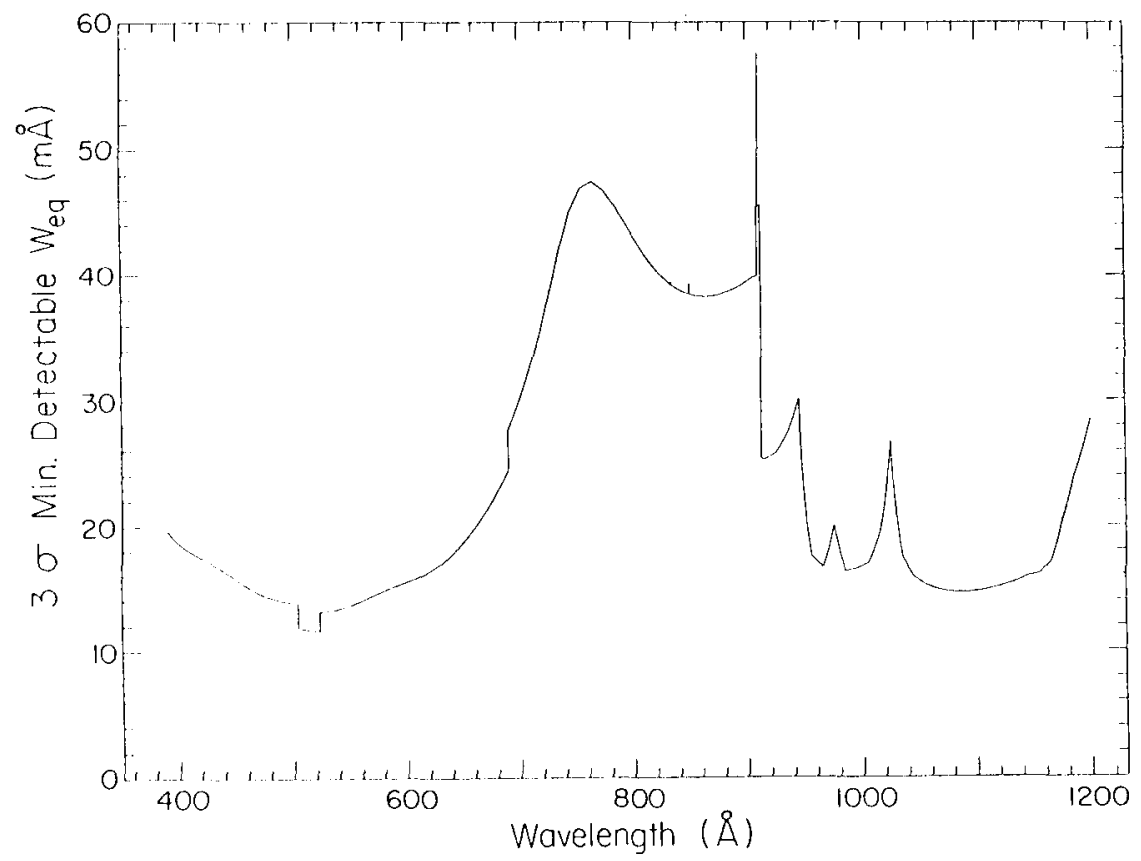

Fig. 4. Minimum equivalent width $(\mathrm{m} \AA$ ) for $3 \sigma$ detection of an absorption line in the spectrum shown in Figure 3 vs. wavelength.

EUVE spectrometer overlaps the ORFEUS bandpass below $\sim 760 \AA$. Its effective area is $1 / 4$ to $1 / 20$ that of ORFEUS at overlap wavelengths. The resolution of the EUVE spectrograph is about 275 , roughly $1 / 30$ that of ORFEUS.

In order to demonstrate the power of this instrument, we have simulated the spectra of a source that is a likely candidate for observation during the ORFEUS mission: a hot WD. Because ORFEUS will provide unique data about the ISM as well as the point sources themselves, we have also estimated the sensitivity of the instrument to detection of absorption line features. This is by no means an exhaustive set of sources we expect to observe, which includes stars from many regions of the $\mathrm{H}-\mathrm{R}$ diagram, interacting binaries, and other celestial objects.

In Figure 3 we show a simulated spectrum of the hot WD HZ 43 as would be observed with this instrument. We display the background-subtracted photon counts per spectral resolution element versus wavelength for an integration time of $3600 \mathrm{~s}$. Discontinuities in the spectrum occur at wavelength breaks between the diffraction gratings and also at the absorption edges of neutral hydrogen and neutral helium. Absorption lines due to the strongest resonance transitions of interstellar neutral hydrogen and neutral helium are shown. These strong absorption features are detected at a high level of significance; it is of interest to evaluate what equivalent width an absorption feature would have that would be detected in this spectrum at some threshold, e.g., $3 \sigma$ significance. We show this minimum detectable equivalent width in Figure 4. Given the measured column density of H I towards HZ 43, we 
expect to detect line features of many elements in a variety of ionization states, greatly improving our understanding of the LISM. Although only a few sources are expected to be as EUV-bright as $\mathrm{HZ} 43$, with longer integration times it will be possible to perform similar studies on a variety of WD targets.

\section{Acknowledgements}

This research was supported in part by NASA grant NGR-003-450.

\section{References}

Davidsen, A.F., Kimble, R.A., Durrance, S.T., Bowers, C.W., and Long, K.S.: 1989, R. F. Malina and S. Bowyer (eds.), Extreme Ultraviolet Astronomy, New York: Pergamon Press

Harada, T. and Kita, T.: 1980, Appl. Opt 19, (23), 3987

Kraemer et al.: 1990, This volume, pp. 177-184

Lampton, M., Siegmund, O.H.W., and Raffanti, R.: 1987, Rev. Sci. Instrum. 58, (12), 2298

Malina, R.F. and Bowyer, S.: 1989, R. F. Malina and S. Bowyer (eds.), Extreme Ultraviolet Astronomy, New York: Pergamon Press

Martin, C., Jelinsky, P., Lampton, M., Malina, R.F., and Anger, H.O.: 1981, Rev. Sci. Instrum. 52, (7), 1067

Siegmund, O.H.W., Everman, E., Vallerga, J.V., and Lampton, M.: 1988, Appl. Opt. 27, (8), 1568 